\title{
Ultra-high Accuracy Microwave Positioning System based on Free Space Frequency Synchronization
}

Q Zhao ( $\sim$ zq18234042973@163.com )

Beijing Institute of Control Engineering

Y.T. Chen

Tsinghua University

\section{Z.B. Wang}

Tsinghua University

L.J. Wang

Tsinghua University

\section{Research Article}

Keywords: Ultra-high Accuracy Microwave Positioning System, Free Space Frequency Synchronization, industrial automation

Posted Date: September 23rd, 2021

DOl: https://doi.org/10.21203/rs.3.rs-888831/v1

License: (c) (1) This work is licensed under a Creative Commons Attribution 4.0 International License. Read Full License 


\title{
Ultra-high accuracy microwave positioning system based on free space frequency synchronization
}

\author{
Q. Zhao ${ }^{1, *}$, Y. T. Chen ${ }^{2,4}$, Z. B. Wang ${ }^{2}$, and L. J. Wang ${ }^{2,3,4}$ \\ ${ }^{1}$ Beijing Institute of Control Engineering, Beijing 100090, China \\ ${ }^{2}$ State Key Laboratory of Precision Measurement Technology and Instruments, Tsinghua University, Beijing 100084, \\ China \\ ${ }^{3}$ Department of Physics, Tsinghua University, Beijing 100084, China \\ ${ }^{4}$ Department of Precision Instrument, Tsinghua University, Beijing 100084, China \\ *zq18234042973@163.com
}

\begin{abstract}
The ultra-high accuracy indoor and outdoor positioning is fundamental for a variety of applications such as industrial automation, Internet of Things and structure monitoring. Approaches based on optical methods, ultrasound and computer vision are often suffer from limited coverage areas, obstruction by objects and high computing load. While the GNSS and conventional radar-like radio frequency (RF) methods can suffer from insufficient accuracy and are not feasible for many scenarios. Now we achieve an inverse GPS microwave positioning system based on ultra-stable frequency synchronization in free space and carrier phase difference principle to surpass the limitation of current methods. The stability of frequency synchronization link is better than $10^{-13} / \mathrm{s}$. The distance resolution retrieved from phase information is 25 micrometres and the Mean Squred Error (MSE) of three-dimensional positioning is 16 micrometres. An ultra-high accuracy positioning system with large coverage area, compatibility and versatility can potentially be achieved gearing to extensive needs.
\end{abstract}

\section{Introduction}

Interest and research in accurate indoor and outdoor positioning are growing recently with a variety of Location Based Services (LBS) such as automatic intelligence, structure monitoring and so on. Many approaches have been proposed but suffer from numerous fundamental limitations ${ }^{1-3}$. Typically, optical methods provide $\mu \mathrm{m}$ to $\mathrm{mm}$ level accuracy but offer limited coverage areas $^{2,4}$. Computer vision systems achieve accuracy levels between tens of $\mu \mathrm{m}$ to $\mathrm{dm}$ but are limited by cost since multiple cameras and sophisticated imaging algorithms are required in three-dimensional (3D) positioning applications ${ }^{5,6}$. Ultrasound ranging and imaging is an alternative approach at $\mathrm{cm}$ level accuracy but is limited to a $10 \mathrm{~m}$ coverage area in terms of impedance matching and strong decay when going through different layers of materials, especially for air gaps ${ }^{7}$. These methods share similar problems in terms of line-of-sight (LoS) obstruction but are vulnerable to metrology and industrial robots if environment and markers are appropriate. Methods based on radio frequency (RF) have also been widely investigated. The RF identification (RFID) system can obtain $\mathrm{dm}$ to $\mathrm{m}$ level accuracy related to the density of tag deployment and reading distance ${ }^{8-10}$. Accuracy at $\mathrm{cm}$ level is possible in the Ultra-Wideband (UWB) system where multi-path interference is severe ${ }^{11-14}$. The Wireless Personal Area Networks (WPANs) including WLAN, ZigBee, Bluetooth and so on are low cost, low power and small size with accuracy of $\mathrm{m}$ level in indoor environments typically cover $20 \mathrm{~m}$ to $30 \mathrm{~m}^{15-17}$. In outdoor positioning, the Global Navigation Satellite System (GNSS) is widely used, providing $\mathrm{cm}$ level accuracy assisted with differential operation and alternatively, in GNSS challenged environments, pseudolite system with $\mathrm{cm}$ level accuracy has been demonstrated ${ }^{18}$. However, challenges remain giving consideration to both ultra-high accuracy and large coverage area, especially considering the compatibility and versatility in complex environments.

The basic measuring principles are common techniques for distance and angular observations ${ }^{19}$. The principle of time of arrival (ToA) is based on measuring the absolute travel time of a signal from a transmitter to a receiver and relies on precise synchronization of transmitter and receiver's clocks. Time difference of arrival (TDoA) has the advantage that a possible receiver's clock bias is not relevant. Any constant time offset of a non-synchronous receiver's clock is eliminated by subtraction. Furthermore, phase difference (PD) uses the received carrier phase to determine the distance between two devices. The phase information of the RF signal is more sensitive to the distance between the transmitter and receiver, and can give higher accuracy if the ambiguity results from frequency synchronization can be resolved ${ }^{20}$.

In this paper, we demonstrate an inverse GPS microwave positioning system based on ultra-stable frequency synchronization with phase compensation in free space. Key aspects are discussed, with particular emphasis on the design of positioning 
scheme and devices. The object to be located is attached with a small signal transmitter and identified by the frequency. Four stand-alone low cost transceivers are used to track and up-convert the signal from object. These transceivers we called " slave stations" play a role as reference nodes or satellites in a traditional GPS scheme. The signals from slave stations are received by a self-made hardware called "main station", where the carrier phase data is processed and coordinate of the object are calculated using phase difference (PD) principle in a host computer. The frequency synchronisation between main station and slave stations is desired since distance variation is retrieved from phase of received signals, which is in a cyclic linear relation with respect to the phase variation at the reference frequency. In the experiment, The frequency stability of synchronisation link is better than $10^{-13} / \mathrm{s}$. The distance resolution retrieved from phase data is 25 micrometres and the Mean Squred Error (MSE) of three-dimensional positioning is 16 micrometres. All frequencies of the system are chose from open band of Industrial Scientific Medical (ISM) for civil applications.

\section{Microwave positioning system design and implementation}

The inverse GPS microwave positioning scheme makes use of phase difference (PD) principle to achieve accurate measurement. To benchmark performance of the scheme, we built a system that consists of a small signal transmitter, four slave stations and a main station as shown in Fig. 1. The transmitter attached to the object sends a sinusoidal signal to slave stations, the frequency of which is $438 \mathrm{MHz}$ with a power below $21 \mathrm{dBm}$. As the picture of slave stations set-up shown in Fig. 2, the received signal after band-pass filter (BPF) and low-noise amplifier (LNA) is then up-converted by the local oscillators (LO) at 2.04, 2.05 , 2.06 and $2.07 \mathrm{GHz}$. The up-converted 2.478, 2.488, 2.498, and 2.508 GHz signals are then retransmitted to main station after amplification and filtering. All slave stations and main station share the same frequency reference. Thus, they can be configured as coherent transceivers. Because of the inverse GPS scheme, the synchronization problem between transmitter of the object and slave stations is also readily avoided. The set-up of main station is shown in Fig. 3. We use a software defined radio (SDR,

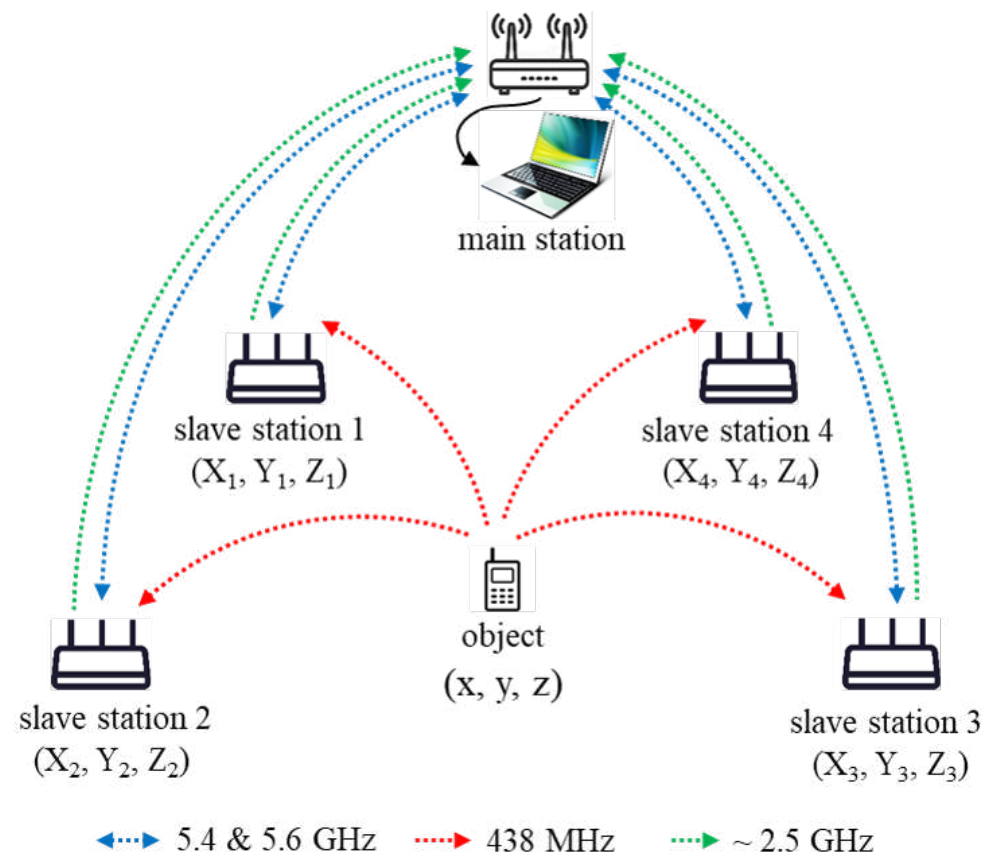

Figure 1. The experimental set-up of the inverse GPS microwave positioning system. The object attached with a small transmitter sends a $438 \mathrm{MHz}$ signal (red links) to slave stations. The slave stations send the up-converted signals at about 2.5 $\mathrm{GHz}$ (green links) to main station and they share the same reference by free space frequency synchronization (blue links). The coordinates of slave stations $\left(X_{i}, Y_{i}, Z_{i}\right)$ is fixed and the coordinate of object $(x, y, z)$ is solved in main station .

Ettus B210) as RF front to receive signals from slave stations. These signals are amplified, filtered and down-converted by the local oscillator (LO) at $2.496 \mathrm{GHz}$ to intermediate frequencies, which are sampled by the analogue-to-digital converter (ADC, AD9361). The digitized intermediate frequency signals are send to the host computer and processed via GNU Radio and Jupyter notebook. Four LO signals at -18, $-8,2$ and $12 \mathrm{MHz}$ are generated by the direct digital synthesizer (DDS) of computer and mixed with digitized intermediate frequency signals correspondingly to calculate carrier phase differences and coordinate of the object. 


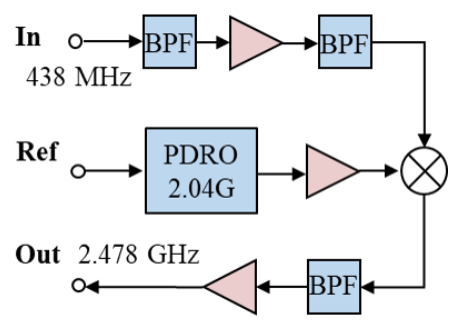

Figure 2. The experimental set-up of slave station with a $2.04 \mathrm{GHz}$ local oscillator. The reference is provided by the free space frequency synchronization link.(BPF: band-pass filter, PDRO: phased-locked dielectric resonator oscillator).

The phase noise is the fundamental limit for our positioning system and the first technique to achieve high resolution is to employ an ultra-stable frequency reference to main station and slave stations ${ }^{21}$. As the experimental set-up shown in Fig. 4, the basic principle of a frequency synchronisation link in free space is mainly described as active phase noise compensation and frequency conversion ${ }^{22}$. It consists of two parts: the local site transmitter in main station and the remote site receivers in slave stations. By detecting and compensating phase noise in the transmission path and a series of frequency conversion, four links disseminate different frequency signals being phase locked to a common reference in main station simultaneously. The uplink and downlink frequencies are $5.4 \mathrm{GHz}$ and $5.6 \mathrm{GHz}$ respectively to avoid interference. The relative stability of a dissemination link is better than $10^{-13} / \mathrm{s}$.

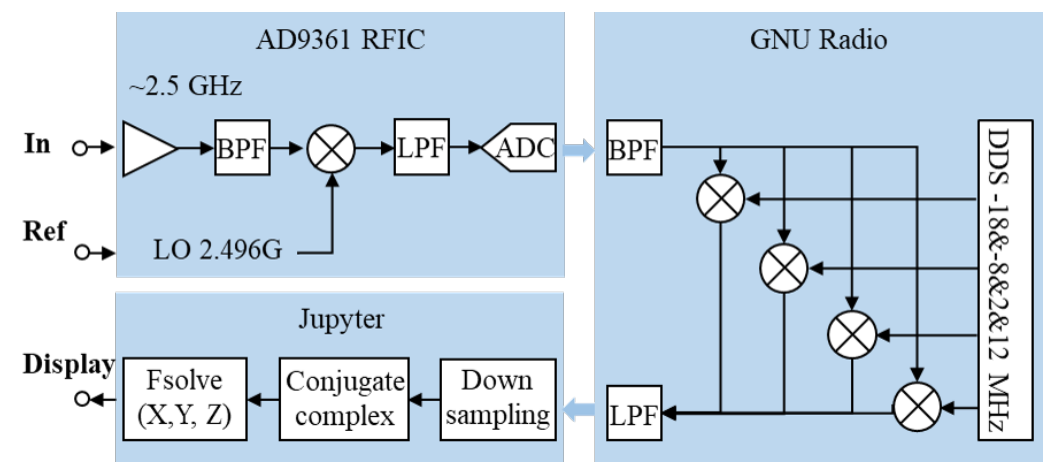

Figure 3. The experimental set-up of main station. The sampling rate of the AD9361 is $48 \mathrm{MHz}$, the down-sampling rate is $10^{2}$ and the moving average window in algorithm is $10^{4}$. The reference is provided by the free space frequency synchronization link. (LPF: low-pass filter, ADC: analogue-to-digital converter).

\section{Positioning experimental results and analyses}

Then a quasi-periodic movement of the object is conducted to investigate the positioning performance. In this inverse GPS and phase difference based scheme, the positioning equations are listed as below:

$$
\left\{\begin{array}{l}
d_{12}(t)+N_{12} \lambda+\Delta l_{12}=f\left(\mathbf{r}(t), \mathbf{r}_{1}\right)-f\left(\mathbf{r}(t), \mathbf{r}_{2}\right) \\
d_{13}(t)+N_{13} \lambda+\Delta l_{13}=f\left(\mathbf{r}(t), \mathbf{r}_{1}\right)-f\left(\mathbf{r}(t), \mathbf{r}_{3}\right) \\
d_{14}(t)+N_{14} \lambda+\Delta l_{14}=f\left(\mathbf{r}(t), \mathbf{r}_{1}\right)-f\left(\mathbf{r}(t), \mathbf{r}_{4}\right)
\end{array}\right.
$$

Here, $\mathbf{r}(t)=(x(t), y(t), z(t))$ is coordinate of the object solved from positioning equations, $\mathbf{r}_{i}=\left(X_{i}, Y_{i}, Z_{i}\right)$ are coordinates of slave stations, $f\left(\mathbf{r}(t), \mathbf{r}_{i}\right)=\sqrt{\left(x(t)-X_{i}\right)^{2}+\left(y(t)-Y_{i}\right)^{2}+\left(z(t)-Z_{i}\right)^{2}}$ is the distance between the object and slave stations, $\lambda$ is the wavelength of transmitter signal, $d_{i j}(t)=\lambda \Delta \phi_{i j}(t) / 2 \pi$ is the distance differences from slave stations to the object derived from carrier phase differences, $N_{i j}$ is integer ambiguity, $\Delta l_{i j}$ is the distance differences from slave stations to main station. The experiment conducted in an open indoor environment. The positions of main station and slave stations are fixed and the coordinates of four slave stations are $(6000 \mathrm{~mm}, 8000 \mathrm{~mm}, 3000 \mathrm{~mm}),(6000 \mathrm{~mm}, 0,3000 \mathrm{~mm}),(0,8000 \mathrm{~mm}, 1000 \mathrm{~mm})$ and $(0,0,3000 \mathrm{~mm})$, correspondingly. The sampling rate of the AD9361 is $48 \mathrm{MHz}$, the down-sampling rate is $10^{2}$ and the moving average window in algorithm is $10^{4}$. The frequency of quasi-periodic movement is about $2 \mathrm{~Hz}$, and the amplitude is about 30 $\mathrm{mm}$. Thus, the integer ambiguity $N_{i j}$ and the distance differences from slave stations to main station $\Delta l_{i j}$ are constants. Solving the equations above and the measurement results are shown in Fig. 5. 


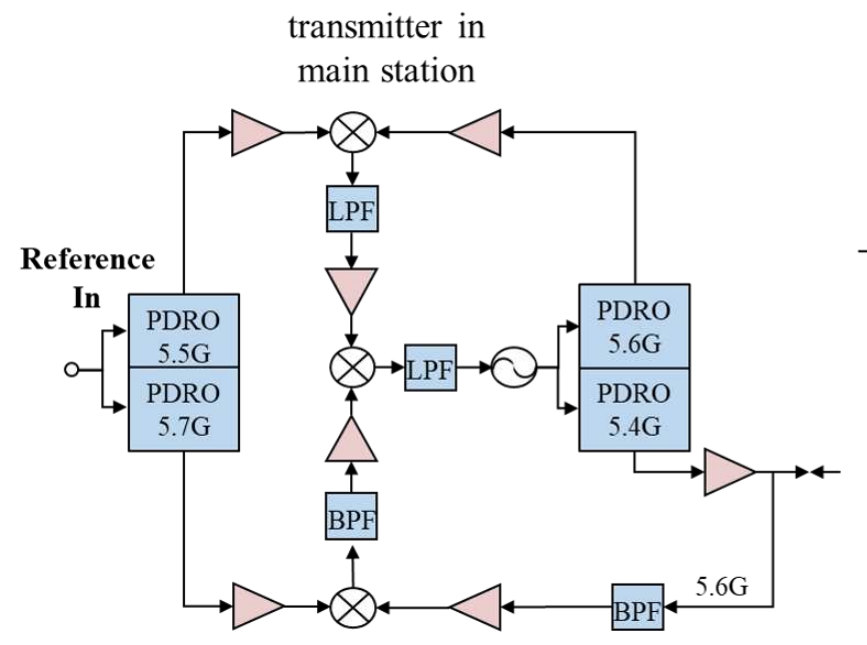

\begin{abstract}
receiver in
\end{abstract}
slave stations

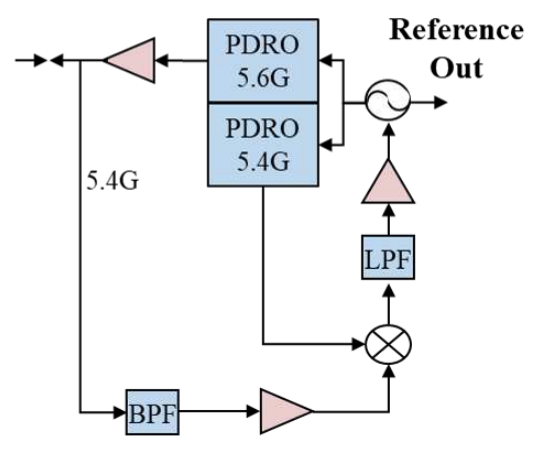

Figure 4. The experimental set-up of the free space frequency synchronization system. The external reference of the synchronization links in main station is provided by an oven controlled crystal oscillator.

Fig. 6 shows the positioning measurement results when the object is fixed. The distance differences from slave stations to object are observed and the resolution is 25 micrometres. The coordinates of this fixed object are calculated and the Mean Squred Error (MSE) of the position is 16 micrometres.

\title{
Discussions
}

The environment of experiment is reasonably controlled to avoid excessive effect. However, phase errors and uncertainties caused by multi-path interference play an important part in positioning accuracy and resolution. High-directivity antennas can be used to reduce the multi-path effect by providing low antenna gain for undesirable directions. The sampling rate of the ADC in SDR, the down-sampling rate and the moving average window configured in algorithm synergistically determine the bandwidth of positioning system according to the Nyquist-Shannon sampling theorem. Thus, when the ambient contain low frequency noise in the channel condition or the positioning system needs to be adapted with broader bandwidth, more sophisticated algorithms should be applied.

In the future, the wavelength integer from cyclic ambiguity and simultaneous multiple objects identification can be resolved by the code division multiple access (CDMA). Moreover, various information of sensors attached to the transmitter can be send to main station using this communication link Some of the Wireless Personal Area Networks, for example, WLAN, ZigBee and Bluetooth working at the same frequency also can be compatible with our system. This way, a multi-source fusion positioning scheme is in prospect.

\section{Conclusions}

We have achieved an inverse GPS microwave positioning system using free space ultra-stable frequency synchronization and phase difference (PD) principle. The relative stability ofrequency synchronization link is better than $10^{-13} / \mathrm{s}$. We measured a quasi-periodic movement and analysed the positioning performance of the system which offers a resolution of less than 25 micrometres and a three-dimensional positioning Mean Squred Error (MSE) of 16 micrometres. A variety of transmitters at open ISM band like devices in WLAN, ZigBee and Bluetooth networks can be perceived and localized and communication links with additional information can be fused. An ultrahigh accuracy positioning system for extensive applications can potentially be achieved in large coverage area.

\section{Methods}

As shown in Fig. 1, the microwave positioning system is implemented based on an inverse GPS scheme consisting of a main station, four slave stations and a transmitter attached to the object. The transmitter sends a signal at $438 \mathrm{MHz}$ to slave stations. The slave stations receive and mix the signal with the respective LO at 2.04, 2.05, 2.06 and 2.07 GHz. Then the up-converted signals at 2.478, 2.488, 2.498 and $2.508 \mathrm{GHz}$ are resend to main station. The main station consists of a SDR Ettus B210 and a host computer. The SDR is controlled by the computer with GNU Radio and is connected to the computer with a USB cable 


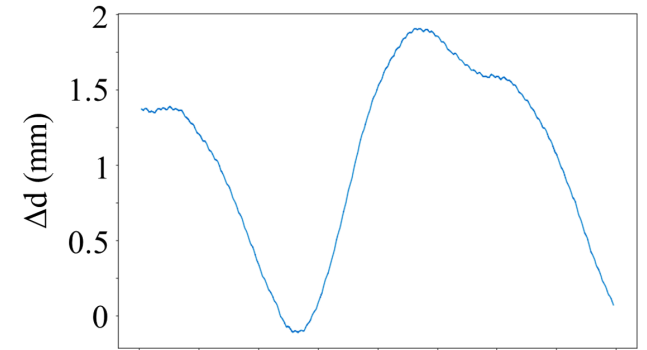

$\begin{array}{lllllllll}0.1 & 0.2 & 0.3 & 0.4 & 0.5 & 0.6 & 0.7 & 0.8 & 0.9\end{array}$

Time (s)

(a) $d_{12}(t)$, the distance differences from slave station 1 and slave station 2 to object.

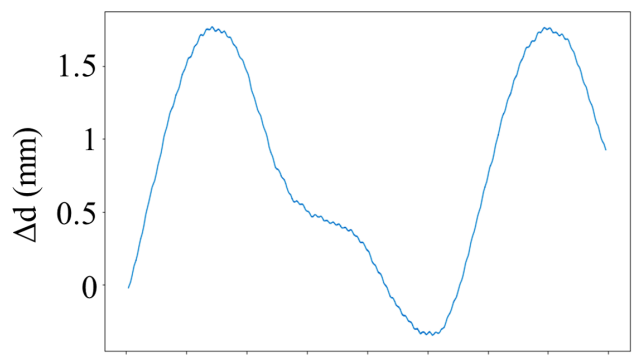

$\begin{array}{lllllllll}0.1 & 0.2 & 0.3 & 0.4 & 0.5 & 0.6 & 0.7 & 0.8 & 0.9\end{array}$

Time (s)

(c) $d_{14}(t)$, the distance differences from slave station 1 and slave station 4 to object.

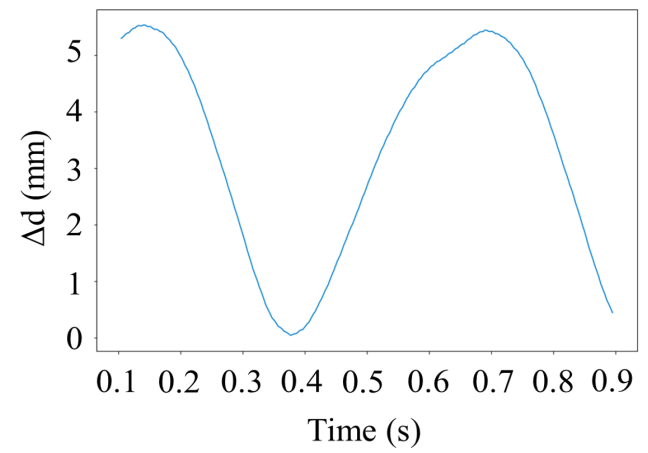

(b) $d_{13}(t)$, the distance differences from slave station 1 and slave station 3 to object.

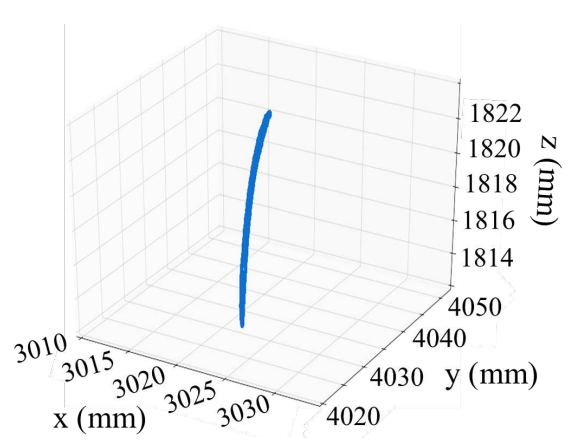

(d) $\mathbf{r}(t)=(x(t), y(t), z(t))$, the coordinate of object solved from the equations.

Figure 5. The positioning measurement results of a quasi-periodic object. The frequency of quasi-periodic movement is about $2 \mathrm{~Hz}$, and the amplitude is about $30 \mathrm{~mm}$.

and serves as a RF front to receive signals from slave stations. In the software, the LO of SDR is set as $2.496 \mathrm{GHz}$, so that the received RF signals mix with LO and generate four intermediate frequency signals. The sampling rate of the AD9361 is configured at $48 \mathrm{MHz}$, then the digitized intermediate frequency signals are send to the host computer and processed via Jupyter notebook. Four LO signals at -18, -8, 2 and $12 \mathrm{MHz}$ are generated by the direct digital synthesizer (DDS) of computer and mixed with digitized intermediate frequency signals correspondingly. The carrier phase differences between slave stations are calculated and the coordinate of object is solved from the positioning equations. All slave stations and main station share the same frequency reference by free space ultra-stable frequency synchronization links. The uplink and downlink frequencies are $5.4 \mathrm{GHz}$ and $5.6 \mathrm{GHz}$ and the relative stability of a dissemination link is better than $10^{-13} / \mathrm{s}$. This way, slave stations are configured as coherent transceivers. The external reference of the synchronization links is provided by an oven controlled crystal oscillator (OCXO, phase noise: $-139 \mathrm{dBc}$ at $100 \mathrm{~Hz},-165 \mathrm{dBc}$ at $1 \mathrm{kHz}$ and $-172 \mathrm{dBc}$ at $10 \mathrm{kHz}$ ). In the experiment, a quasi-periodic moving object and a fixed object are measured and analysed. The surroundings of the set-up are reasonably controlled to avoid very strong microwave reflections, multipath effect and excessive movement of people.

\section{References}

1. Xiaonan, H. \& Edwin, C. K. Radio ranging with ultrahigh resolution using a harmonic radio-frequency identification system. Nat. Electron. 2, 125-131 (2019).

2. Mautz, R. Indoor positioning technologies, DOI: https://doi.org/10.3929/ethz-a-007313554 (2012).

3. Liu, G. \& Shi, L. An overview about development of indoor navigation and positioning technology. J. Navig. Position. 6, 7-14 (2018).

4. Kai, L., Andreas, N., Joachim, H. \& Hartmut, S. High-speed laser localization for mobile robots. Robotics Auton. Syst. 51, 275-296 (2005).

5. Davison, A. J., Reid, I. D. \& Molton, N. D. Monoslam: Real-time single camera slam. IEEE Trans 29, 1052-1067 (2007). 


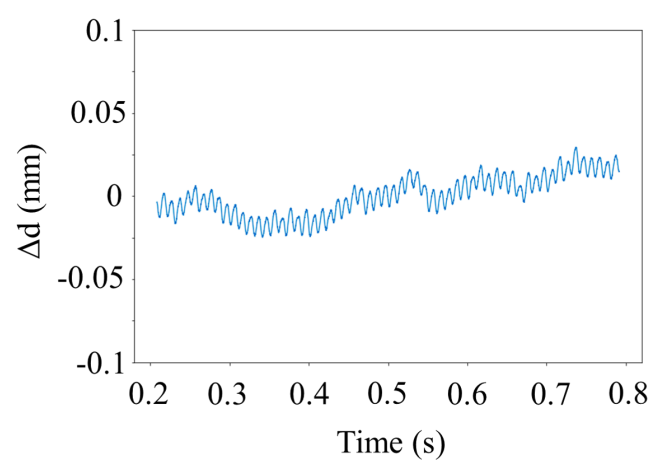

(a) $d_{12}(t)$, the distance differences from slave station 1 and slave station 2 to object.

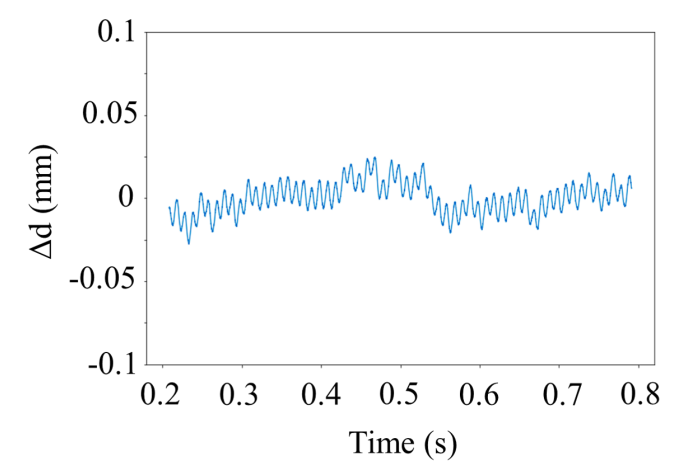

(c) $d_{14}(t)$, the distance differences from slave station 1 and slave station 4 to object.

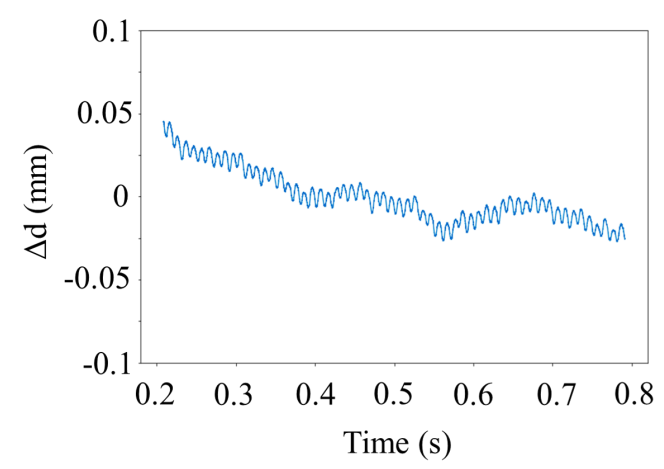

(b) $d_{13}(t)$, the distance differences from slave station 1 and slave station 3 to object.

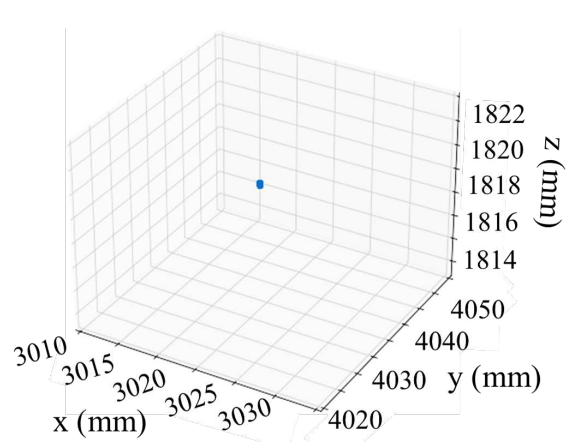

(d) $\mathbf{r}(t)=(x(t), y(t), z(t))$, the coordinate of object solved from the equations.

Figure 6. The positioning measurement results of a fixed object. The distance differences resolution from slave stations to object is 25 micrometres. The Mean Squred Error (MSE) of the position is 16 micrometres.

6. Sergi, F., Guillem, A. \& Carme, T. Lock-in time-of-flight (tof) cameras: a survey. IEEE Sens. J 11, 1917-1926 (2011).

7. Huang, H. \& Gartner, G. A survey of mobile indoor navigation systems. Springer: Heidelberg, Ger. 305-319 (2007).

8. Qiu, L., Huang, Z. \& Liang, X. Survey of indoor location-aware technology for rfid tag. Appl. Res. Comput. 34, 3521-3526 (2017).

9. Omer, M. \& Tian, G. Y. Indoor distance estimation for passive uhf rfid tag based on rssi and rcs. Measurement 127, 425-430 (2018).

10. Hui, X., Ma, Y. \& Kan, E. C. Real-time $3 d$ robotic arm tracking in indoor environment by rf nonlinear backscattering. Proc. ACM 3-5 (2016).

11. Ravikrishnan, H. Ultra-wideband position tracking on an assembly line (2014).

12. Liu, H., Darabi, H., Banerjee, P. \& Liu, J. Localization via ultra-wideband radios: A look at positioning aspects for future sensor networks. IEEE Signal Process. 22, 70-84 (2005).

13. Prete, M. D., Decarli, N., Masotti, D., Dardari, D. \& Costanzo, A. Exploitation of multi-sine intermodulation for passive backscattering uwb localization. Proc. IEEE MTT-S Int. Microw. Symp 262-265 (2018).

14. Nils, P. et al. Radar measurements with micrometer accuracy and nanometer stability using an ultra-wideband 80 ghz radar system. IEEE Top. Conf. on Wirel. Sensors Sens. Networks (WiSNet) 31-33, DOI: https://doi.org/10.1109/WiSNet.2013. 6488624 (2013).

15. Liu, H., Darabi, H., Banerjee, P. \& Liu, J. Survey of wireless indoor positioning techniques and systems. IEEE Trans 37, 1087-1080 (2007).

16. Akyildiz, I. F. \& Su, W. Y. Wireless sensor networks: A survey. Comput. Networks 38, 393-422 (2002).

17. Vasisht, D., Kumar, S. \& Katabi, D. Decimeter-level localization with a single wifi access point. Proc. 13th Usenix Conf. Networked Syst. Des. Implementation 165-178 (2016). 
18. Joel, B., Chris, R. \& Jinling, W. High precision indoor and outdoor positioning using locatanet. J. Glob. Position. Syst. 2, DOI: https://doi.org/10.5081/jgps.2.2.73 (2003).

19. Guoyi, X., Pragya, S. \& Edwin, C. K. Indoor object sensing using radio-frequency identification with inverse solutions. IEEE SENSORS 1-4, DOI: https://doi.org/10.1109/SENSORS47125.2020.9278594 (2020).

20. Piotrowsky, L., Jaeschke, T., Kuppers, S. \& Pohl, N. An unambiguous phase-based algorithm for single-digit micron accuracy distance measurements using fmcw radar. IEEE MTT-S Int. Microw. Symp. (IMS) 552-555, DOI: https: //doi.org/10.1109/MWSYM.2019.8700889 (2019).

21. Scherr, S., Ayhan, M. P. \& Zwick, T. Accuracy limits of a k-band fmcw radar with phase evaluation. 9th Eur. Radar Conf. 246-249 (2012).

22. Miao, J., Wang, B., Gao, C. \& Wang, L. J. Ultra-stable radio frequency dissemination in free space. Rev. Sci. Instruments 84, 104703 (2013).

\section{Author contributions statement}

Q. Zhao and Y. T. Chen conceived the experimental concept; All contributed to the design and Q. Zhao conducted the experiment; Q. Zhao analysed the data; Q. Zhao, Z. B. Wang and L. J. Wang wrote the paper. 